\title{
Joint Ego-Motion and Road Geometry Estimation
}

\author{
Christian Lundquist ${ }^{\mathrm{a}}$, Thomas B. Schön ${ }^{\mathrm{a}}$ \\ ${ }^{a}$ Division of Automatic Control, Department of Electrical Engineering, \\ Linköping University, SE-581 83 Linköping, Sweden
}

\begin{abstract}
We provide a sensor fusion framework for solving the problem of joint ego-motion and road geometry estimation. More specifically we employ a sensor fusion framework to make systematic use of the measurements from a forward looking radar and camera, steering wheel angle sensor, wheel speed sensors and inertial sensors to compute good estimates of the road geometry and the motion of the ego vehicle on this road. In order to solve this problem we derive dynamical models for the ego vehicle, the road and the leading vehicles. The main difference to existing approaches is that we make use of a new dynamic model for the road. An extended Kalman filter is used to fuse data and to filter measurements from the camera in order to improve the road geometry estimate. The proposed solution has been tested and compared to existing algorithms for this problem, using measurements from authentic traffic environments on public roads in Sweden. The results clearly indicate that the proposed method provides better estimates.
\end{abstract}

Key words: sensor fusion, single track model, bicycle model, extended Kalman filter, road geometry estimation.

\section{Introduction}

We are in this paper concerned with the problem of integrated ego-motion and road geometry estimation using information from several sensors. The sensors used to this end are a forward looking camera and radar, together with inertial sensors, a steering wheel sensor and wheel speed sensors. The solution is obtained by casting the problem within an existing sensor fusion framework. An important part of this solution is the nonlinear state-space model. The state-space model contains the dynamics of the ego vehicle, the road geometry, the leading vehicles and the measurement relations. It can then be written in the form

$$
\begin{aligned}
\mathbf{x}_{k+1} & =f\left(\mathbf{x}_{k}, \mathbf{u}_{k}\right)+\mathbf{w}_{k}, \\
\mathbf{y}_{k} & =h\left(\mathbf{x}_{k}, \mathbf{u}_{k}\right)+\mathbf{e}_{k},
\end{aligned}
$$

where $\mathbf{x}_{k} \in \mathbb{R}^{n_{x}}$ denotes the state vector, $\mathbf{u}_{k} \in \mathbb{R}^{n_{u}}$ denotes the input signals, $\mathbf{y}_{k} \in \mathbb{R}^{n_{y}}$ denotes the measurements, $\mathbf{w}_{k} \in \mathbb{R}^{n_{w}}$ and $\mathbf{e}_{k} \in \mathbb{R}^{n_{e}}$ denote the process and measurement noise, respectively. The process model equations, describing the evolution of the state over time are denoted by $f: \mathbb{R}^{n_{x}} \times \mathbb{R}^{n_{u}} \rightarrow \mathbb{R}^{n_{x}}$. Furthermore, the measurement model describing how the measurements

Email addresses: lundquist@isy. liu.se (Christian Lundquist), schon@isy.liu.se (Thomas B. Schön)

Preprint submitted to Information Fusion from the vision system, the radar and the inertial sensors relate to the state is given by $h: \mathbb{R}^{n_{x}} \times \mathbb{R}^{n_{u}} \rightarrow \mathbb{R}^{n_{y}}$. When we have a model in the form (1) we have transformed the problem into a standard nonlinear state estimation problem, where the task is to compute estimates of the state based on the information in the measurements. There are many different ways of solving this problem and we will in this work make use of the popular Extended Kalman Filter (EKF), described in e.g., $[1,2,3]$.

The problem studied in this paper is by no means new, it is the proposed solution that is new. For some early, still very interesting and relevant work on this problem we refer to $[4,5]$. From the camera we can produce estimates of the road geometry based on measurements of the lane markings. This problem is by now rather mature, see e.g., the survey [6] and the recent book [7] for solid accounts. The next step in the development was to make use of the radar information as well. Using radar measurements we can track the leading vehicles, that is, we can estimate the position and velocity of the leading vehicles. Under the assumption that the leading vehicles drive on the same road as the ego vehicle, their positions contain valuable information about the road geometry. This idea was introduced by $[8,9,10]$ and has been further refined in [11, 12]. The combination of radar and vision as well as the advantages and July 1, 2010 
disadvantages of these sensors are discussed in [13, 14]. Furthermore, the ego vehicle model in $[13,14]$ is comparable with the one used in the present work. The four wheel speeds are used to estimate the path of the ego vehicle, which unlike the present work is separated from the leading vehicles dynamics and the lane estimate.

The leading vehicles are used to improve the road geometry in the present work; however the opposite is also possible as the recent work $[15,16]$ shows, where the vehicle detection algorithm benefits from the lane information. Vision and radar are used in [15], whereas vision and lidar are used in [16]. In [17] lidar is used to detect the leading vehicle, and the movement of the leading vehicle is then used to estimate the lane and the driven path, which in turn is used to autonomously follow this vehicle. This works well even for curved and narrow roads. Unmarked and winding rural roads may be hard to detect, recent research in this area is presented in [18], where stereo vision and image radar are used within a marginalized particle filter to obtain 3D information and improve the task of lane recognition. Information obtained from road-side structures may be used to improve the estimate of the lane shape and the position of the vehicle within the lane, as showed in [19], where only a monocular camera is used. Furthermore, at construction sites it is hard to identify the temporary lanes, a method for this using color images and beacon extraction is presented in [20]. In [21] the authors present an algorithm for free space estimation, capable of handling non-planar roads, using a stereo camera system.

Lane tracking has also been tackled using radar sensors, see e.g., [22, 23, 24, 25] and laser sensors, see e.g. [26]. There have been several approaches making use of reflections from the road boundary, such as crash barriers and reflection posts, to compute information about the free space, see e.g. [27, 28, 29] for some examples using laser scanners and [30], where radar is used.

To summarize, our approach is able to improve the performance by making use of a dynamic model of the ego vehicle and a new dynamic model of the road at the same time as we make use of the motion of the leading vehicles. The new road process model describes the curvature of the ego vehicle's currently driven path. This should be compared with existing road models, used in most of the publications mentioned above, where the road's curvature is modeled according to road construction standards. The advantage of our new road model is that we are able to directly include information of the ego vehicles motion into the estimate of the road geometry.
In the subsequent section we provide a brief introduction to the sensor fusion framework we work with and explain how the present problem fits into this framework. An essential part of this framework is the dynamical model (1a), which is derived in Section 3. Furthermore, the corresponding measurement model (1b) is introduced in Section 4. In Section 5 the proposed solution is evaluated using measurements from real and relevant traffic environments from public roads in Sweden. Finally, the conclusions are given in Section 6. For convenience we provide a list of the relevant notation in the appendix.

\section{Sensor Fusion}

In order to successfully solve the problem under study in this work it is imperative to have a good understanding of sensor fusion. Sensor fusion is defined as the process of using information from several different sensors to compute an estimate of the state of a dynamical system.

We need a dynamic model and a measurement model in the form (1) in order to be able to produce an estimate of the state. These models are derived in detail in Section 3 and Section 4. However, for the sake of the present discussion we will briefly discuss the model here. The state vector $\mathbf{x}_{k}$ consists of three parts according to

$$
\mathbf{x}_{k}=\left[\begin{array}{c}
\mathbf{x}_{\mathrm{E}, k} \\
\mathbf{x}_{\mathrm{R}, k} \\
\mathbf{x}_{\mathrm{T}, k}
\end{array}\right],
$$

where $\mathbf{x}_{\mathrm{E}, k}$ denotes the state of the ego vehicle, $\mathbf{x}_{\mathrm{R}, k}$ denotes the state of the road and $\mathbf{x}_{\mathrm{T}, k}$ denotes the state of one leading vehicle (also referred to as a target). In deriving the evolution of these states over time we will end up with continuous-time differential equations in the form

$$
\dot{\mathbf{x}}(t)=g(\mathbf{x}(t), \mathbf{u}(t))
$$

However, according to (1) we required the model to be in discrete time. The simplest way of obtaining a difference equation from (3) is to make use of the standard forward Euler method, which approximates (3) at time $t$ according to

$$
\mathbf{x}(t+T)=\mathbf{x}(t)+T g(\mathbf{x}(t), \mathbf{u}(t)) \triangleq f\left(\mathbf{x}_{t}, \mathbf{u}_{t}\right),
$$

where $T$ denotes the sample time. The measurement model is of course already in discrete time.

The estimate of the state is computed by a state estimator of some kind. This state estimator makes use of 
the measurements from the different sensors to produce an estimate of the so called filtering probability density function (pdf) $p\left(\mathbf{x}_{k} \mid \mathbf{y}_{1: k}\right)$, where $\mathbf{y}_{1: k} \triangleq\left\{\mathbf{y}_{i}\right\}_{i=1}^{k}$ denotes all the measurements from time 1 to time $k$. This density function contains all there is to know about the state $\mathbf{x}_{k}$, given the information in the measurements $\mathbf{y}_{1: k}$. Once an approximation of $p\left(\mathbf{x}_{k} \mid \mathbf{y}_{1: k}\right)$ is available it can be used to form many different estimates and the most commonly used estimate is the conditional mean estimate

$$
\hat{\mathbf{x}}_{k \mid k}=\mathrm{E}\left(\mathbf{x}_{k} \mid \mathbf{y}_{1: k}\right)
$$

This estimate will be used in the present work as well.

Since we are looking for an algorithm capable of working in real-time it is important to understand how the filtering pdf evolves over time. Now, it is wellknown (see e.g., [31]) that a sequential solution can be obtained according to

$$
\begin{aligned}
p\left(\mathbf{x}_{k} \mid \mathbf{y}_{1: k}\right) & =\frac{p\left(\mathbf{y}_{k} \mid \mathbf{x}_{k}\right) p\left(\mathbf{x}_{k} \mid \mathbf{y}_{1: k-1}\right)}{\int p\left(\mathbf{y}_{k} \mid \mathbf{x}_{k}\right) p\left(\mathbf{x}_{k} \mid \mathbf{y}_{1: k-1}\right) d \mathbf{x}_{k}}, \\
p\left(\mathbf{x}_{k+1} \mid \mathbf{y}_{1: k}\right) & =\int p\left(\mathbf{x}_{k+1} \mid \mathbf{x}_{k}\right) p\left(\mathbf{x}_{k} \mid \mathbf{y}_{1: k}\right) d \mathbf{x}_{k} .
\end{aligned}
$$

Here, it is also worth mentioning that since we have assumed additive noise in the model (1), we have explicit expressions for $p\left(\mathbf{x}_{k+1} \mid \mathbf{x}_{k}\right)$ and $p\left(\mathbf{y}_{k} \mid \mathbf{x}_{k}\right)$ according to

$$
\begin{aligned}
p\left(\mathbf{x}_{k+1} \mid \mathbf{x}_{k}\right) & =p_{\mathbf{w}_{k}}\left(\mathbf{x}_{k+1}-f\left(\mathbf{x}_{k}, \mathbf{u}_{k}\right)\right), \\
p\left(\mathbf{y}_{k} \mid \mathbf{x}_{k}\right) & =p_{\mathbf{e}_{k}}\left(\mathbf{y}_{k}-h\left(\mathbf{x}_{k}, \mathbf{u}_{k}\right)\right),
\end{aligned}
$$

where $p_{\mathbf{w}_{k}}(\cdot)$ and $p_{\mathbf{e}_{k}}(\cdot)$ denote the pdf's for the process and the measurement noise, respectively.

In the special case, where the equations in the model (1) are linear and the noise is Gaussian, the multidimensional integrals in (6) allows for an analytical solution, the Kalman filter [32]. For a derivation of this kind, see e.g., [33]. However, the problem is that for the general nonlinear, non-Gaussian case that we are facing, there does not exist any closed form solution to (6). Hence, we are forced to make approximations of some kind. The most commonly used approximation is provided by the extended Kalman filter (EKF). The idea underlying the EKF is very simple, approximate the nonlinear model with a linear model subject to Gaussian noise and apply the Kalman filter to this approximation. For a solid account of the EKF we refer to $[3,34]$. Lately the so called particle filter, introduced in [35], has become increasingly popular. This filter often provides a better solution, but it typically requires much more computational effort. For the present application the EKF provides an approximation that is good enough. For a more thorough account of the framework for nonlinear estimation briefly introduced above we refer to [33].

Before we end our brief overview on the sensor fusion problem it is important to stress that a successful sensor fusion framework will, besides the modeling and filtering parts mentioned above, rely on a certain surrounding infrastructure. This surrounding infrastructure deals with issues such as time synchronization between the various sensors, calibration, sensor-near signal processing, track handling, etc. This part of the framework should not be overlooked and a solid treatment of the provided infrastructure is accounted for in [36] for the problem at hand. Despite this it is worth mentioning that the leading vehicles are incorporated into the estimation problem using rather standard techniques from target tracking, such as nearest neighbor data association and track counters in order to decide when to stop tracking a certain vehicle, etc. These are all important parts of the system we have implemented, but it falls outside the scope of this paper and since the techniques are rather standard we simply refer to the general treatments given in e.g., [37, 38].

\section{Dynamic Models}

As mentioned in the introduction our sensor fusion framework needs a state-space model describing the dynamics of the ego vehicle, the road and the leading vehicles. In this section we will derive the differential equations describing the motion of the ego vehicle (Section 3.2), the road (Section 3.3) and the leading vehicles (Section 3.4), also referred to as targets. Finally, in Section 3.5 we summarize these equations and form the process model of the state-space model. However, before we embark on deriving these equations we introduce the overall geometry and some necessary notation in Section 3.1.

\subsection{Geometry and Notation}

The coordinate frames describing the ego vehicle and one leading vehicle are defined in Figure 1. The inertial world reference frame is denoted by $W$ and its origin is $O_{W}$. The ego vehicle's coordinate frame $E$ is located in the center of gravity (CoG). Furthermore, $V_{n}$ is associated to the observed leading vehicle $n$, with $O_{V}$ at the vision and radar sensor of the ego vehicle. Finally, $T_{n}$ is also associated with the observed and tracked leading vehicle $n$, but its origin $O_{T_{n}}$ is located at the leading vehicle. In this work we will use the planar coordinate transformation matrix

$$
R^{W E}=\left[\begin{array}{cc}
\cos \psi_{E} & -\sin \psi_{E} \\
\sin \psi_{E} & \cos \psi_{E}
\end{array}\right]
$$




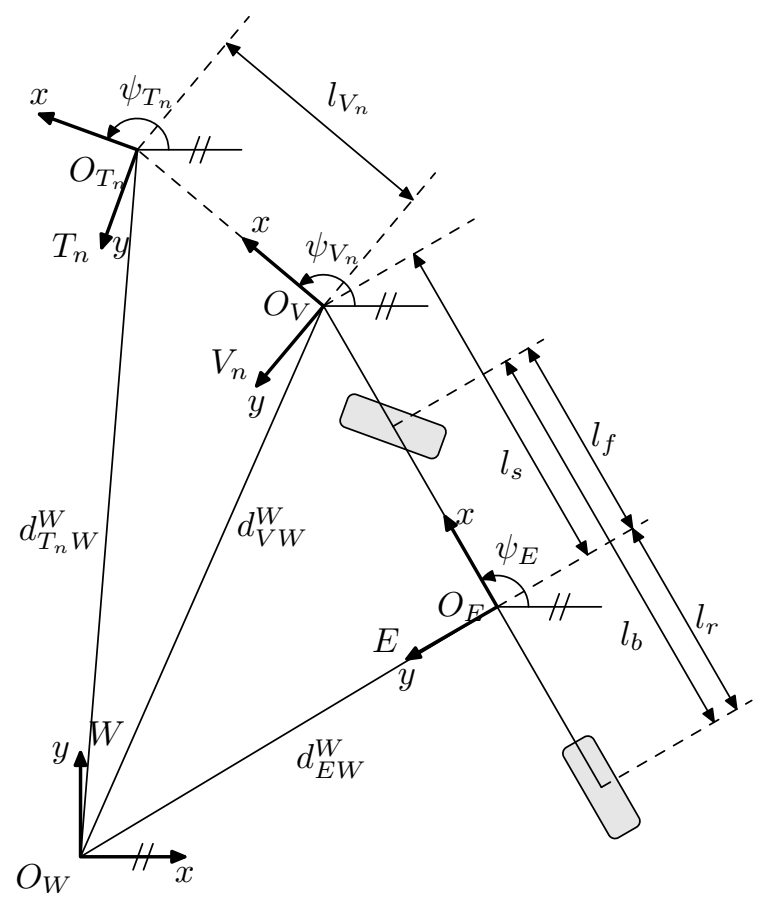

Figure 1: Coordinate frames describing the ego vehicle, with center of gravity in $O_{E}$ and the radar and camera sensors mounted in $O_{V}$. One leading vehicle is positioned in $O_{T_{n}}$.

to transform a vector, represented in $E$, into a vector, represented in $W$, where the yaw angle of the ego vehicle $\psi_{E}$ is the angle of rotation from $W$ to $E$. The geometric displacement vector $d_{E W}^{W}$ is the direct straight line from $O_{W}$ to $O_{E}$ represented with respect to the frame $W$. Velocities are defined as the movement of a frame $E$ relative to the inertial reference frame $W$, but typically resolved in the frame $E$, for example $v_{x}^{E}$ is the velocity of the $E$ frame in its $x$-direction. The same convention holds for the acceleration $a_{x}^{E}$. In order to simplify the notation we leave out $E$ when referring to the ego vehicle's velocity and acceleration. This notation will be used when referring to the various coordinate frames. However, certain frequently used quantities will be renamed, in the interest of readability. The measurements are denoted using superscript $m$. Furthermore, the notation used for the rigid body dynamics is in accordance with [39].

\subsection{Ego Vehicle}

We will only be concerned with the ego vehicle motion during normal driving situations and not at the adhesion limit. This implies that the single track model is sufficient for the present purposes. This model is also referred to as the bicycle model, see e.g., [40, 41] for a solid treatment. The geometry of the single track model with slip angles is shown in Figure 2. It is here worth to point out that the velocity vector of the ego vehicle is typically not in the same direction as the longitudinal axis of the ego vehicle. Instead the vehicle will move along a path at an angle $\beta$ with the longitudinal direction of the vehicle. Hence, the angle $\beta$ is defined as,

$$
\tan \beta=\frac{v_{y}}{v_{x}},
$$

where $v_{x}$ and $v_{y}$ are the ego vehicle's longitudinal and lateral velocity components, respectively. This angle $\beta$ is referred to as the float angle [42] or the vehicle body side slip angle [43].

The slip angle $\alpha_{i}$ is defined as the angle between the central axis of the wheel and the path along which the wheel moves. The phenomenon of side slip is mainly due to the lateral elasticity of the tire. For reasonably small slip angles, at maximum $3 \mathrm{deg}$, it is a good approximation to assume that the lateral friction force of the tire $F_{i}$ is proportional to the slip angle,

$$
F_{i}=C_{\alpha i} \alpha_{i}
$$

The parameter $C_{\alpha i}$ is called cornering stiffness and describes the cornering behavior of the tire. The load transfer to the front axle when braking or to the outer wheels when driving trough a curve influences the parameter value. A model considering these influences is given in [44].

Following this brief introduction to the ego vehicle geometry, we are now ready to give an expression describing the evolution of yaw angle $\psi_{E}$ and the float angle $\beta$ over time

$$
\begin{array}{r}
\ddot{\psi}_{E}=\beta \frac{-C_{\alpha f} l_{f} \cos \delta_{f}+C_{\alpha r} l_{r}}{I_{z z}} \\
-\dot{\psi}_{E} \frac{C_{\alpha f} l_{f}^{2} \cos \delta_{f}+C_{\alpha r} l_{r}^{2}}{I_{z z} v_{x}}+\frac{C_{\alpha f} l_{f} \tan \delta_{f}}{I_{z z}}, \\
\dot{\beta}=-\beta \frac{C_{\alpha f} \cos \delta_{f}+C_{\alpha r}+\dot{v}_{x} m}{m v_{x}} \\
-\dot{\psi}_{E}\left(1+\frac{C_{\alpha f} l_{f} \cos \delta_{f}-C_{\alpha r} l_{r}}{v_{x}^{2} m}\right)+\frac{C_{\alpha f} \sin \delta_{f}}{m v_{x}},
\end{array}
$$

where $m$ denotes the mass of the vehicle and $I_{z z}$ denotes the moment of inertia of the vehicle about its vertical axis in the center of gravity. These single track model equations are well-known in the literature, see e.g., [43]. 


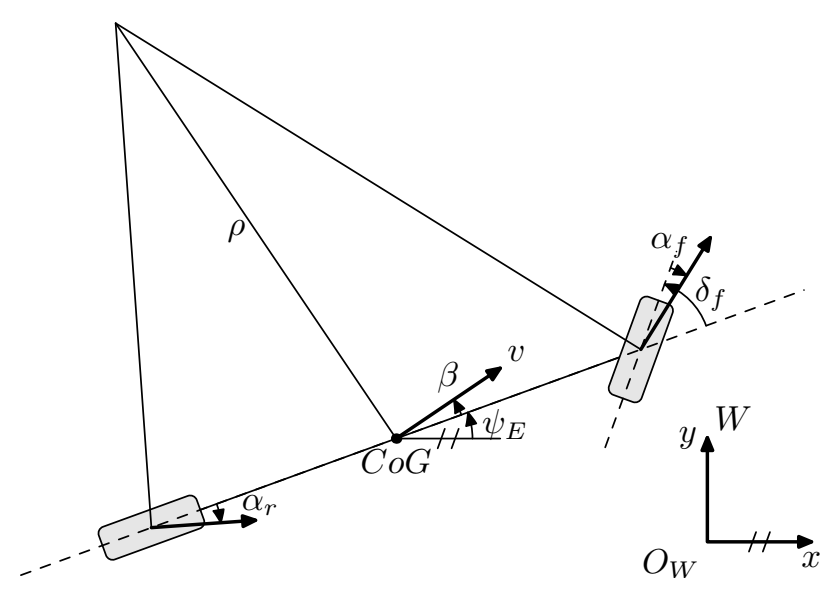

Figure 2: In the single track model the wheels on each axle are modeled as single units. The velocity vector $v$, with the float angle $\beta$ to the longitudinal axis of the vehicle, is attached at the center of gravity. Furthermore, the wheel slip angles are referred to as $\alpha_{f}$ and $\alpha_{r}$. The front wheel angle is denoted by $\delta_{f}$ and the current radius is denoted by $\rho$.

\subsection{Road Geometry}

We start this section by defining the road variables and expressing a typical way to parameterize a road. The section is continued with a derivation of a new model for the road that makes use of the dynamic motion of the ego vehicle.

\subsubsection{Background}

The most essential component in describing the road geometry is the curvature $c$, which we will define as the curvature of the white lane marking to the left of the ego vehicle. An overall description of the road geometry is given in Figure 3. The heading angle $\psi_{R}$ is defined as the tangent of the road at the level of the ego vehicle in the world reference frame $W$, see Figure 4 . The angle $\delta_{r}$ is the angle between the tangent of the road curvature and the longitudinal axis of the ego vehicle. Note that this angle can be measured by sensors mounted on the ego vehicle. Furthermore, we define $\delta_{R}$ as

$$
\delta_{R} \triangleq \delta_{r}-\beta
$$

i.e., the angle between the ego vehicles direction of motion (velocity vector) and the road curvature tangent.

The road curvature $c$ is typically parameterized according to

$$
c\left(x_{c}\right)=c_{0}+c_{1} x_{c}
$$

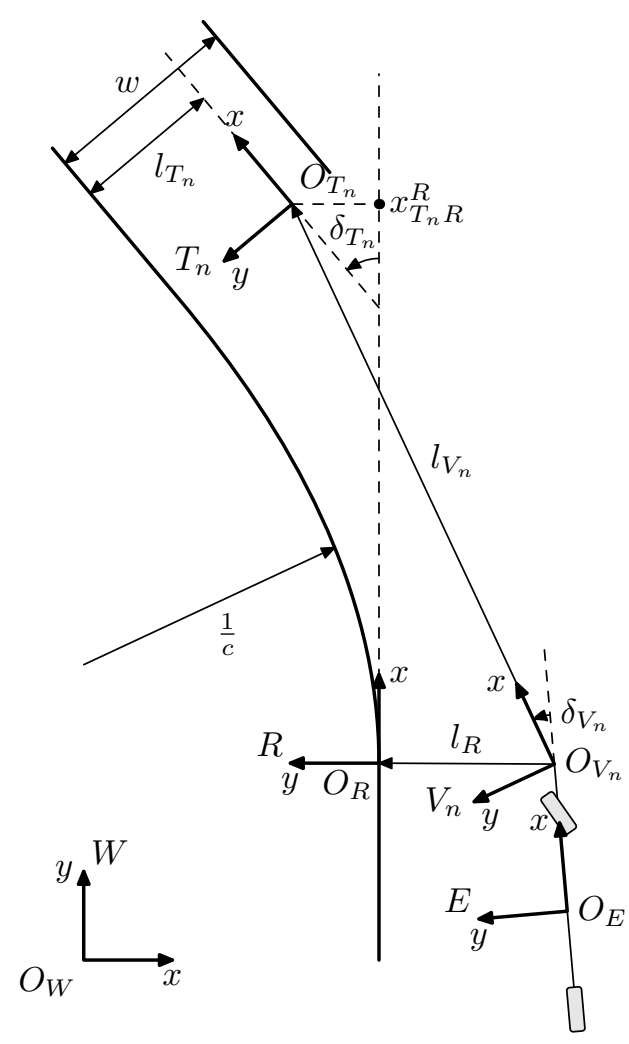

Figure 3: Relations between one leading vehicle in $O_{T_{n}}$, the ego vehicle and the road. The distance between the ego vehicle's longitudinal $x$-axis and the white lane to its left is $l_{R}(t)$. The leading vehicle's distance to the lane marking is $l_{T_{n}}$ and its heading angle in the road frame $R$ is $\delta_{T_{n}}$. The lane width is $w$.

where $x_{c}$ is the position along the road in a road aligned coordinate frame and $x_{c}=0$ at the vehicles center of gravity. Furthermore, $c_{0}$ describes the local curvature at the ego vehicle position and $c_{1}$ is the distance derivative (hence, the rate of change) of $c_{0}$. It is common to make use of a road aligned coordinate frame when deriving an estimator for the road geometry, a good overview of this approach is given in [12]. There are several advantages using road aligned coordinate frames, particularly the motion models of the other vehicles on the same road can be greatly simplified. However, the flexibility of the motion models is reduced and basic dynamic relations such as Newton's and Euler's laws cannot be directly applied. Since we are using a single track model of the ego vehicle, we will make use of a Cartesian coordinate frame. A good polynomial approximation of the shape of the road curvature is given by

$$
y^{E}=l_{R}+x^{E} \tan \delta_{r}+\frac{c_{0}}{2}\left(x^{E}\right)^{2}+\frac{c_{1}}{6}\left(x^{E}\right)^{3},
$$




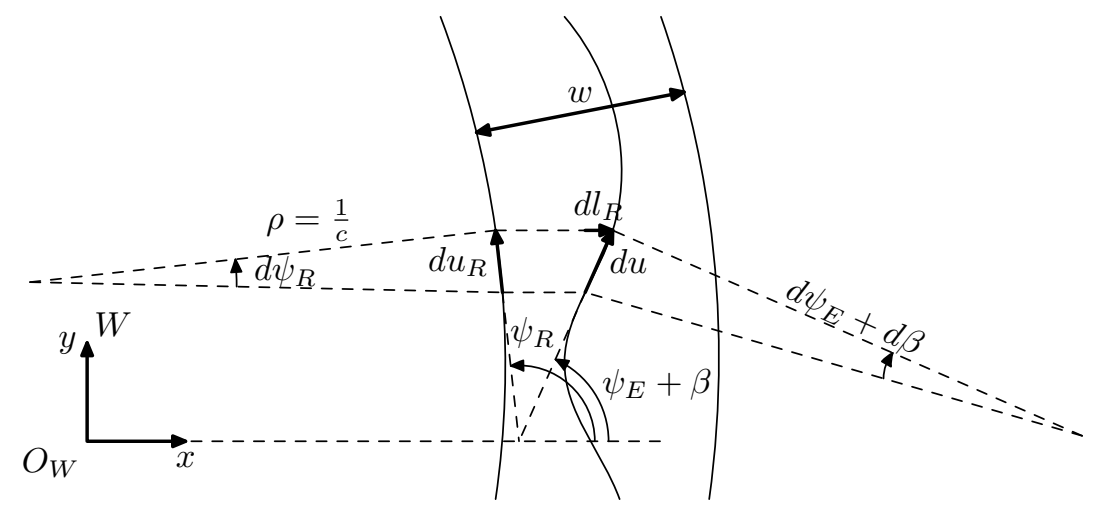

Figure 4: Infinitesimal segments of the road curvature $d u_{R}$ and the driven path $d u$ are shown together with the angles $\delta_{R}=\psi_{R}-\left(\psi_{E}+\beta\right)$.

where $l_{R}(t)$ is defined as the time dependent distance between the ego vehicle and the lane marking to the left, see e.g., $[5,12]$.

The following dynamic model is often used for the road

$$
\begin{aligned}
& \dot{c}_{0}=v c_{1}, \\
& \dot{c}_{1}=0,
\end{aligned}
$$

which can be interpreted as a velocity dependent integration. It is interesting to note that (15) reflects the way in which roads are commonly built [5]. However, we will now derive a new dynamic model for the road, that makes use of the road geometry introduced above.

\subsubsection{A New Dynamic Road Model}

Assume that $d u_{R}$ is an infinitesimal part of the road curvature or an arc of the road circle with the angle $d \psi_{R}$, see Figure 4. A segment of the road circle can be described as

$$
d u_{R}=\frac{1}{c_{0}} d \psi_{R},
$$

which after division with the infinitesimal change in time $d t$ is given by

$$
\frac{d u_{R}}{d t}=\frac{1}{c_{0}} \frac{d \psi_{R}}{d t} .
$$

Assuming that the left hand side can be reformulated according to

$$
\frac{d u_{R}}{d t}=v_{x} \cos \left(\psi_{R}-\psi_{E}\right) \approx v_{x}
$$

this yields

$$
v_{x}=\frac{1}{c_{0}} \dot{\psi}_{R} .
$$

The angle $\psi_{R}$ can be expressed as

$$
\psi_{R}=\psi_{E}+\beta+\delta_{R},
$$

by rewriting (12). Re-ordering equation (19) and using the derivative of (20) to substitute $\dot{\psi}_{R}$ yields

$$
\dot{\delta}_{R}=c_{0} v_{x}-\left(\dot{\psi}_{E}+\dot{\beta}\right)
$$

which by substituting $\dot{\beta}$ with (11b) according to

$$
\begin{aligned}
\dot{\delta}_{R}=c_{0} v_{x} & -\beta \frac{-C_{\alpha f} \cos \delta_{f}-C_{\alpha r}-\dot{v}_{x} m}{m v_{x}} \\
& +\dot{\psi}_{E} \frac{C_{\alpha f} l_{f} \cos \delta_{f}-C_{\alpha r} l_{r}}{v_{x}^{2} m}-\frac{C_{\alpha f} \sin \delta_{f}}{m v_{x}}
\end{aligned}
$$

results in a differential equation of the road angle $\delta_{R}$. A similar relation has been used in $[5,45]$.

We also need a differential equation for the road curvature, which can be found by differentiating (21) w.r.t. time,

$$
\ddot{\delta}_{R}=\dot{c}_{0} v_{x}+c_{0} \dot{v}_{x}-\ddot{\psi}_{E}-\ddot{\beta}
$$

From the above equation we have

$$
\dot{c}_{0}=\frac{\ddot{\delta}_{R}+\ddot{\psi}_{E}+\ddot{\beta}-c_{0} \dot{v}_{x}}{v_{x}}
$$

Let us assume that $\ddot{\delta}_{R}=0$. Furthermore, differentiating $\dot{\beta}$, from (11b), w.r.t. time and inserting this together with $\ddot{\psi}_{E}$, given in (11a), into the above expression yields the 
differential equation

$$
\begin{gathered}
\dot{c}_{0}=\frac{1}{\left(I_{z z} m^{2} v_{x}\right)^{4}}\left(C_{\alpha r}^{2}\left(I_{z z}+l_{r}^{2} m\right)\left(-\dot{\psi}_{E} l_{r}+\beta v_{x}\right)\right. \\
\quad+C_{\alpha f}^{2}\left(I_{z z}+l_{f}^{2} m\right)\left(\dot{\psi}_{E} l_{f}+\left(\beta-\delta_{f}\right) v_{x}\right) \\
+C_{\alpha r} I_{z z} m\left(-3 \dot{\psi}_{E} \dot{v}_{x} l_{r}+3 \beta \dot{v}_{x} v_{x}+\dot{\psi}_{E} v_{x}^{2}\right) \\
\quad+\dot{v}_{x} I_{z z} m^{2} v_{x}\left(2 \beta \dot{v}_{x}+v_{x}\left(\dot{\psi}_{E}-c_{0} v_{x}\right)\right) \\
+C_{\alpha f}\left(C_{\alpha r}\left(I_{z z}+l_{r}\left(-l_{f}\right) m\right)\left(\dot{\psi}_{E} l_{b}-2 \dot{\psi}_{E} l_{r}+2 \beta v_{x}-\delta_{f} v_{x}\right)\right. \\
\left.\left.+I_{z z} m\left(3 \dot{\psi}_{E} \dot{v}_{x} l_{f}+\left(3 \beta-2 \delta_{f}\right) \dot{v}_{x} v_{x}+\left(\dot{\delta}_{f}+\dot{\psi}_{E}\right) v_{x}^{2}\right)\right)\right)
\end{gathered}
$$

for the road curvature.

In this model $c_{0}$ is defined at the ego vehicle and thus describes the currently driven curvature, whereas for the curvature described by the state-space model (15) and by the polynomial (13) it is not entirely obvious where $c_{0}$ is defined.

Finally, we need a differential equation describing how the distance $l_{R}(t)$ between the ego vehicle and the lane markings changes over time. Assume again an infinitesimal arc $d u$ of the circumference describing the ego vehicle's curvature. By contemplating Figure 4 we have

$$
d l_{R}=d u \sin \delta_{R},
$$

where $\delta_{R}$ is the angle between the ego vehicle's velocity vector and the road. Dividing this equation with an infinitesimal change in time $d t$ and using $d u / d t=v$ yield the differential equation

$$
\dot{l}_{R}=v_{x} \sin \left(\delta_{R}+\beta\right),
$$

which concludes the derivation of the road geometry model.

\subsection{Leading Vehicles}

The leading vehicles are also referred to as targets $\mathrm{T}_{n}$ The coordinate frame $T_{n}$ moving with target $n$ has its origin located in $O_{T_{n}}$, as we previously saw in Figure 3. It is assumed that the leading vehicles are driving on the road, quite possibly in a different lane. More specifically, it is assumed that they are following the road curvature and thus that their heading is in the same direction as the tangent of the road.

For each target $\mathrm{T}_{n}$, there exists a coordinate frame $V_{n}$, with its origin $O_{V}$ at the position of the sensor. Hence, the origin is the same for all targets, but the coordinate frames have different heading angles $\psi_{V_{n}}$. This angle, as well as the distance $l_{V_{n}}$, depend on the targets position in space. From Figure 3 it is obvious that,

$$
d_{E W}^{W}+d_{V_{n} E}^{W}+d_{T_{n} V}^{W}-d_{T_{n} W}^{W}=0,
$$

or more explicitly,

$$
\begin{gathered}
x_{E W}^{W}+l_{s} \cos \psi_{E}+l_{V_{n}} \cos \psi_{V_{n}}-x_{T_{n} W}^{W}=0, \\
y_{E W}^{W}+l_{s} \sin \psi_{E}+l_{V_{n}} \sin \psi_{V_{n}}-y_{T_{n} W}^{W}=0 .
\end{gathered}
$$

Let us now define the relative angle to the leading vehicle as

$$
\delta_{V_{n}} \triangleq \psi_{V_{n}}-\psi_{E}
$$

It is worth noticing that this angle can be measured by a sensor mounted on the vehicle.

The target $\mathrm{T}_{n}$ is assumed to have zero lateral velocity in the $V_{n}$ frame, i.e., $\dot{y}^{V_{n}}=0$, since it is always fixed to the $x^{V_{n}}$-axis. If we transform this relation to the world frame $W$, using the geometry of Figure 1 we have

$$
R^{V W} \cdot \dot{d}_{T_{n} W}^{W}=\left[\begin{array}{l}
\cdot \\
0
\end{array}\right],
$$

where the top equation of the vector equality is nondescriptive and the bottom equation can be rewritten as

$$
-\dot{x}_{T_{n} W}^{W} \sin \psi_{V_{n}}+\dot{y}_{T_{n} W}^{W} \cos \psi_{V_{n}}=0 .
$$

The velocity vector of the ego vehicles is applied in the center of gravity $O_{E}$. The derivative of (29) is used together with the velocity components of the ego vehicle and (32) to get an expression for the derivative of the relative angle to the leading vehicle w.r.t. time according to

$$
\left(\dot{\delta}_{V_{n}}+\dot{\psi}_{E}\right) l_{V_{n}}+\dot{\psi}_{E} l_{s} \cos \delta_{V_{n}}+v_{x} \sin \left(\beta-\delta_{V_{n}}\right)=0 .
$$

This equation is rewritten, forming the differential equation

$$
\dot{\delta}_{V_{n}}=-\frac{\dot{\psi}_{E} l_{s} \cos \delta_{V_{n}}+v_{x} \sin \left(\beta-\delta_{V_{n}}\right)}{l_{V_{n}}}-\dot{\psi}_{E}
$$

of the relative angle $\dot{\delta}_{V_{n}}$ to the leading vehicles.

\subsection{Summarizing the Dynamic Model}

The state-space models derived in the previous sections are nonlinear and they are given in continuous time. Hence, in order to make use of these equations in the EKF we will first linearize them and then make use of (4) in order to obtain a state-space model in discrete time according to (1). This is a rather standard procedure, see e.g., [46, 47]. At each time step $k$, the nonlinear state-space model is linearized by evaluating the Jacobian (i.e., the partial derivatives) of the $f\left(\mathbf{x}_{k}, \mathbf{u}_{k}\right)$ matrix introduced in (4) at the current estimate $\hat{\mathbf{x}}_{k \mid k}$. It is worth noting that this Jacobian is straightforwardly 
computed off-line using symbolic or numerical software, such as Mathematica. Hence, we will not go through the details here. However, for future reference we will briefly summarize the continuous-time dynamic model here.

In the final state-space model the three parts (ego vehicle, road and leading vehicles) of the dynamic model are augmented, resulting in a state vector of dimension $6+4 \cdot$ (Number of leading vehicles). Hence, the size of the state vector varies with time, depending on the number of leading vehicles that are tracked at a specific instance of time.

The ego vehicle model is described by the following states,

$$
\mathbf{x}_{\mathrm{E}}=\left[\begin{array}{lll}
\dot{\psi}_{E} & \beta & l_{R}
\end{array}\right]^{T},
$$

i.e., the yaw rate, the float angle and the distance to the left lane marking. The front wheel angle $\delta_{f}$, which is calculated from the measured steering wheel angle, and the ego vehicle longitudinal velocity $v_{x}$ and acceleration $\dot{v}_{x}$ are modeled as input signals,

$$
\mathbf{u}_{k}=\left[\begin{array}{lll}
\delta_{f} & v_{x} & \dot{v}_{x}
\end{array}\right]^{T} .
$$

The nonlinear state-space model $\dot{\mathbf{x}}_{\mathrm{E}}=g_{\mathrm{E}}(\mathbf{x}, \mathbf{u})$ is given by

$$
\begin{gathered}
g_{\mathrm{E}}(\mathbf{x}, \mathbf{u})= \\
{\left[\begin{array}{c}
\beta \frac{-C_{\alpha f} l_{f} \cos \delta_{f}+C_{\alpha r} l_{r}}{I_{z z}}-\dot{\psi}_{E} \frac{C_{\alpha f} l_{f}^{2} \cos \delta_{f}+C_{\alpha r} l_{r}^{2}}{I_{z z} v_{x}}+\frac{C_{\alpha f} l_{f} \tan \delta_{f}}{I_{z z}} \\
-\beta \frac{C_{\alpha f} \cos \delta_{f}+C_{\alpha r}+\dot{v}_{x} m}{m v_{x}}-\dot{\psi}_{E}\left(1+\frac{C_{\alpha f} l_{f} \cos \delta_{f}-C_{\alpha r} l_{r}}{v_{x}^{2} m}\right)+\frac{C_{\alpha f} \sin \delta_{f}}{m v_{x}} \\
v_{x} \sin \left(\delta_{R}+\beta\right)
\end{array}\right] .}
\end{gathered}
$$

\section{(37) 4. Measurement Model}

The corresponding differential equations were previously given in (11a), (11b) and (27), respectively.

The states describing the road $\mathbf{x}_{\mathrm{R}}$ are the road curvature $c_{0}$ at the ego vehicle position, the angle $\delta_{R}$ between the ego vehicles direction of motion and the road curvature tangent and the width of the lane $w$, i.e.,

$$
\mathbf{x}_{\mathrm{R}}=\left[\begin{array}{lll}
c_{0} & \delta_{R} & w
\end{array}\right]^{T} .
$$

The differential equations for $c_{0}$ and $\delta_{R}$ were given in (25) and (22), respectively. When it comes to the width of the current lane $w$, we have

$$
\dot{w}=0 \text {, }
$$

motivated by the fact that $w$ does not change as fast as the other variables, i.e., the nonlinear state-space model

$$
\begin{aligned}
& \dot{\mathbf{x}}_{\mathrm{R}}=g_{\mathrm{R}}(\mathbf{x}, \mathbf{u}) \text { is given by } \\
& g_{\mathrm{R}}(\mathbf{x}, \mathbf{u})= \\
& {\left[\begin{array}{c}
\dot{c}_{0} \\
c_{0} v_{x}+\beta \frac{C_{\alpha f} \cos \delta_{f}+C_{\alpha r}+\dot{x}_{x} m}{m v_{x}}+\dot{\psi} \frac{C_{\alpha f} l_{f} \cos \delta_{f}-C_{\alpha r} l_{r}}{v_{x}^{2} m}-\frac{C_{\alpha f} \sin \delta_{f}}{m v_{x}} \\
0
\end{array}\right] .}
\end{aligned}
$$

A target is described by the following states, azimuth angle $\delta_{V_{n}}$, lateral position $l_{T_{n}}$ of the target, distance between the target and the ego vehicle $l_{V_{n}}$ and relative velocity between the target and the ego vehicle $\dot{l}_{V_{n}}$. Hence, the state vector is given by

$$
\mathbf{x}_{\mathrm{T}}=\left[\begin{array}{llll}
\delta_{V_{n}} & l_{T_{n}} & i_{V_{n}} & l_{V_{n}}
\end{array}\right]^{T} .
$$

The derivative of the azimuth angle was given in (34). It is assumed that the leading vehicle's lateral velocity is small, implying that $\dot{l}_{T_{n}}=0$ is a good assumption (compare with Figure 3). Furthermore, it can be assumed that the leading vehicle accelerates similar to the ego vehicle, thus $\ddot{l}_{V_{n}}=0$ (compare with e.g., [12]). The statespace model $\dot{\mathbf{x}}_{\mathrm{T}}=g_{\mathrm{T}}(\mathbf{x}, \mathbf{u})$ of a leading vehicle (target) is

$$
g_{\mathrm{T}}(\mathbf{x}, \mathbf{u})=\left[\begin{array}{c}
-\frac{\dot{\psi}_{E} l_{s} \cos \delta_{V_{n}}+v_{x} \sin \left(\beta-\delta_{V_{n}}\right)}{l_{V_{n}}}-\dot{\psi}_{E} \\
0 \\
0 \\
\dot{l}_{V_{n}}
\end{array}\right] .
$$

Note that the dynamic models given in this section are nonlinear in $\mathbf{u}$.

The measurement model (1b) describes how the measurements $\mathbf{y}_{k}$ relates to the state variables $\mathbf{x}_{k}$. In other words, it describes how the measurements enter the estimator. We will make use of superscript $m$ to denote measurements. Let us start by introducing the measurements relating directly to the ego vehicle motion, by defining

$$
\mathbf{y}^{1}=\left[\begin{array}{ll}
\dot{\psi}_{E}^{m} & a_{y}^{m}
\end{array}\right]^{T},
$$

where $\dot{\psi}_{E}^{m}$ and $a_{y}^{m}$ are the measured yaw rate and the measured lateral acceleration, respectively. They are both measured with the ego vehicle's inertial sensor in the center of gravity (CoG). The ego vehicle lateral acceleration in the $\mathrm{CoG}$ is

$$
a_{y}=v_{x}\left(\dot{\psi}_{E}+\dot{\beta}\right)+\dot{v}_{x} \beta \text {. }
$$


By replacing $\dot{\beta}$ with the expression given in (11b) and at the same time assuming that $\dot{v}_{x} \beta \approx 0$ we obtain

$$
\begin{aligned}
a_{y} & =v_{x}\left(\dot{\psi}_{E}+\dot{\beta}\right) \\
& =-\beta \frac{C_{\alpha f} \cos \delta_{f}+C_{\alpha r}+m \dot{v}_{x}}{m} \\
& +\dot{\psi}_{E} \frac{-C_{\alpha f} l_{f} \cos \delta_{f}+C_{\alpha r} l_{r}}{m v_{x}}+\frac{C_{\alpha f}}{m} \sin \delta_{f} .
\end{aligned}
$$

From this it is clear that the measurement of the lateral acceleration contains information about the ego vehicle states. Hence, the measurement equation corresponding to (43) is given by

$$
\begin{aligned}
& h^{1}= \\
& {\left[\begin{array}{c}
\dot{\underline{\psi}}_{E} \\
-\beta \frac{C_{\alpha f} \cos \delta_{f}+C_{\alpha r}+m \dot{v}_{x}}{m}+\dot{\psi}_{E} \frac{-C_{\alpha f} l_{f} \cos \delta_{f}+C_{\alpha r} l_{r}}{m v_{x}}+\frac{C_{\alpha f}}{m} \sin \delta_{f}
\end{array}\right] .}
\end{aligned}
$$

The vision system provides measurements of the road geometry and the ego vehicle position on the road according to

$$
\mathbf{y}^{2}=\left[\begin{array}{llll}
c_{0}^{m} & \delta_{r}^{m} & w^{m} & l_{R}^{m}
\end{array}\right]^{T}
$$

and the corresponding measurement equations are given by

$$
h^{2}=\left[\begin{array}{llll}
c_{0} & \left(\delta_{R}+\beta\right) & w & l_{R}
\end{array}\right]^{T} .
$$

An obvious choice would have been to use the state $\delta_{r}$, instead of the sum $\delta_{R}+\beta$, however, we have chosen to split these since we are interested in estimating both of these quantities.

In order to include measurements of a leading vehicle we require that it is detected both by the radar and the vision system. The range $l_{V_{n}}$ and the range rate $\dot{l}_{V_{n}}$ are measured by the radar. The azimuth angle is also measured by the radar, but not used directly in this framework. Instead, the accuracy of the angle estimate is improved by using the camera information. We will not describe these details here, since it falls outside the scope of this work. The corresponding measurement vector is

$$
\mathbf{y}^{3}=\left[\begin{array}{lll}
\delta_{V_{n}}^{m} & l_{V_{n}}^{m} & l_{V_{n}}^{m}
\end{array}\right]^{T}
$$

Since these are state variables, the measurement equation is obviously

$$
h^{3}=\left[\begin{array}{lll}
\delta_{V_{n}} & \dot{l}_{V_{n}} & l_{V_{n}}
\end{array}\right]^{T} .
$$

The fact that the motion of the leading vehicles reveals information about the road geometry allows us to make use of their motion in order to improve the road geometry estimate. This will be accomplished by introducing a nontrivial artificial measurement equation according to

$h^{4}=l_{R}+\left(\delta_{R}+\beta\right) l_{V_{n}} \cos \delta_{V_{n}}+\frac{c_{0}}{2}\left(l_{V_{n}} \cos \delta_{V_{n}}\right)^{2}+\frac{l_{T_{n}}}{\cos \delta_{T_{n}}}$,

which is derived from Figure 3 and describes the predicted lateral distance of a leading vehicle in the ego vehicles coordinate frame $E$. In order to model the road curvature we introduce the road coordinate frame $R$, with its origin $O_{R}$ on the white lane marking to the left of the ego vehicle. This implies that the frame $R$ is moving with the frame $E$ of the ego vehicle. The angle $\delta_{T_{n}} \triangleq \psi_{T_{n}}-\psi_{R}$ is derived by considering the road's slope at the position of the leading vehicle, i.e.,

$$
\delta_{T_{n}}=\arctan \frac{d y^{R}}{d x^{R}}=\arctan c_{0} x^{R},
$$

where $x^{R}=x_{T_{n} R}^{R}$, see Figure 3. The Cartesian $x$ coordinate of the leading vehicle $\mathrm{T}_{n}$ in the $R$-frame is

$$
x_{T_{n} R}^{R}=x_{T_{n} E}^{E}-l_{s} \approx l_{V_{n}} \frac{\cos \delta_{V_{n}}}{\cos \delta_{r}} .
$$

The sensors only provide range $l_{V_{n}}^{m}$ and azimuth angle $\delta_{V_{n}}^{m}$. Hence, the corresponding quasi-measurement is

$$
\mathbf{y}^{4}=l_{V_{n}}^{m} \sin \left(\delta_{V_{n}}^{m}\right),
$$

describing the measured lateral distance to a leading vehicle in the ego vehicle's coordinate frame. This might seem a bit ad hoc at first. However, the validity of the approach has recently been justified in the literature, see e.g., [48].

\section{Experiments and Results}

The experiments presented in this section are based on measurements acquired on public roads in Sweden during normal traffic conditions. The test vehicle is a Volvo S80 equipped with a forward looking $77 \mathrm{GHz}$ mechanically scanning FMCW radar and a forward looking vision sensor (camera), measuring the distances and angles to the targets. The image sensor includes object and lane detection and provides for example the lane curvature. Information about the ego vehicle motion, such as the steering wheel angle, yaw rate, etc. were acquired directly from the CAN bus.

Before stating the main results in this section we outline how to estimate the parameters of the ego vehicle and how the filter is tuned. Subsequently we state the 

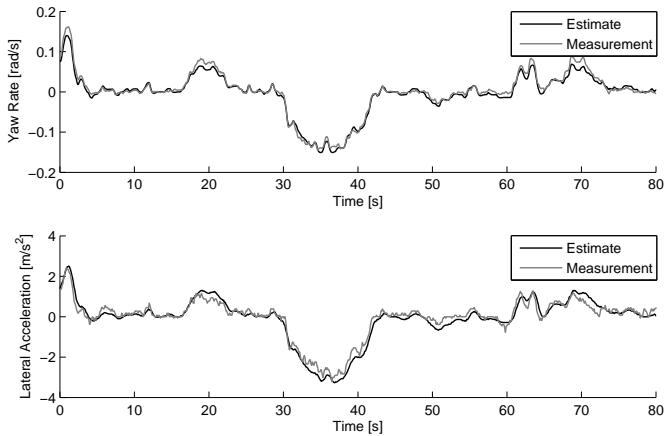

Figure 5: Comparing the simulated result of the nonlinear state-space model (black) with measured data (gray) of a validation data set. The upper plot shows the yaw rate and the lower shows the lateral acceleration.

results of the ego vehicle validation. We compare our road curvature estimates with two other sensor fusion approaches as well as one road model.

\subsection{Parameter Estimation and Filter Tuning}

Most of the ego vehicle's parameters, such as the dimensions, the mass and the moment of inertia were provided by the vehicle manufacturer. Since the cornering stiffness is a parameter which describes the properties between road and tire it has to be estimated for the given set of measurements. An on-line method to estimate the cornering stiffness parameter using recursive least square is presented in [44]. However, in the present work an exhaustive search was accomplished off-line using a batch of measurements to estimate $C_{\alpha f}$ and $C_{\alpha r}$. A state-space model with the differential equations given in (11a) and (11b) and with the yaw rate $\dot{\psi}_{E}$ and the float angle $\beta$ in the state vector was used for this purpose. Furthermore, the front wheel angle $\delta_{f}$ and the ego vehicle longitudinal velocity $v_{x}$ were modeled as input signals. The measurements were provided by the yaw rate $\dot{\psi}_{E}^{m}$ and the lateral acceleration $a_{y}^{m}$. The corresponding measurement equation was given in (46). The data used to identifying the cornering stiffness parameters was split into two parts, one estimation part and one validation part. This facilitates cross-validation, where the parameters are estimated using the estimation data and the quality of the estimates can then be assessed using the validation data [49].

The approach is further described in [50]. The resulting state-space model with the estimated parameters was validated using the validation data and the result is given in Figure 5.
The process and measurement noise covariances are the design parameters in the extended Kalman filter (EKF). It is assumed that the covariances are diagonal and that there are no cross correlations between the measurement noise and the process noise. The present filter has ten states and ten measurement signals, which implies that 20 parameters have to be tuned. The tuning was started using physical intuition of the error in the process equations and the measurement signals. In a second step, the covariance parameters were tuned simply by trying to minimize the root mean square error (RMSE) of the estimated $\hat{c}_{0}$ and the reference curvature $c_{0}$. The estimated curvature was obtained by running the filter using the estimation data set. The calculation of the reference value is described in [51]. The chosen design parameters were validated on a different data set and the results are discussed in the subsequent sections.

\subsection{Validation Using Ego Vehicle Signals}

The state variables of the ego vehicle are according to (35), the yaw rate, the float angle and the distance to the left lane marking. The estimated and the measured yaw rate signals are, as expected, very similar. As described in Section 5.1, the parameters of the vehicle model were optimized with respect to the yaw rate, hence it is no surprise that the fusion method decreases the residual further. A measurement sequence acquired on a rural road is shown in Figure 6a. Note that the same measurement sequence is used in Figures 5 to 7, which will make it easier to compare the estimated states.

The float angle $\beta$ is estimated, but there is no reference or measurement signal to compare it to. An example is shown in Figure $6 \mathrm{~b}$. For velocities above $30-40 \mathrm{~km} / \mathrm{h}$, the float angle appears more or less like the mirror image of the yaw rate, and by comparing with Figure 6a, we can conclude that the sequence is consistent.

The measurement signal of the distance to the left white lane marking $l_{R}^{m}$ is produced by the vision system OLR (Optical Lane Recognition). Bad lane markings or certain weather conditions can cause errors in the measurement signal. The estimated state $l_{R}$ of the fusion approach is very similar to the pure OLR signal.

\subsection{Road Curvature Estimation}

An essential idea with the sensor fusion approach introduced in this paper is to make use of the single track ego vehicle model in order to produce better estimates of the road curvature. In this section we will compare this approach to approaches based on other models of the ego vehicle and the road geometry. 


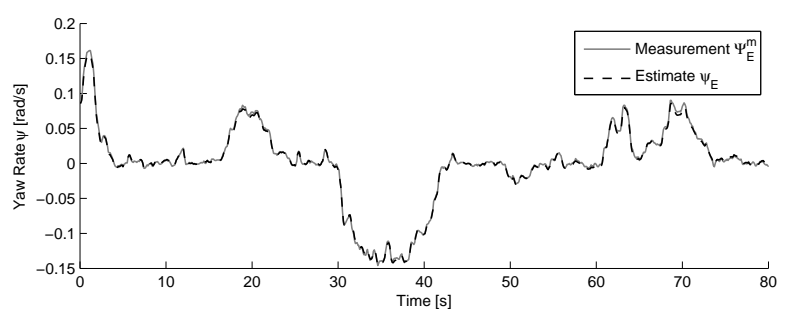

(a)

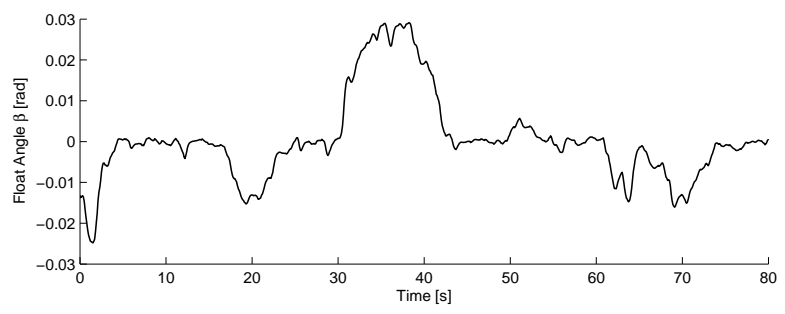

(b)

Figure 6: A comparison between the ego vehicle's measured (gray) and estimated yaw rate (black dashed) using the sensor fusion approach in this paper is shown in (a). The estimated float angle $\beta$ for the same data sequence is shown in (b).

Fusion 1 is the sensor fusion approach shown in this paper.

Fusion 2 is a similar approach, thoroughly described in [12]. An important difference to fusion 1 is that the ego vehicle is modeled with a constant velocity model, which is less complex. The float angle $\beta$ is not estimated. Furthermore, the road is modeled according to (15) and a road aligned coordinate frame is used. This method is similar to the approaches used in e.g., $[8,9,10]$.

Fusion 3 comprehends the ego vehicle model of fusion 1 and the road model of fusion 2, i.e., substituting (25) by (15) and introducing the seventh state $c_{1}$. Furthermore, a Cartesian coordinate frame is used. This method, but without considering the leading vehicles is similar to the ones described in e.g., [5] and [52].

Model is the ego vehicle and road state-space model given in this paper, described by the motion models (37) and (40) and the measurement models (46) and (48), without the extended Kalman filter.

The curvature estimate $\hat{c}_{0}$ from the sensor fusion approaches, the model and the raw measurement from the optical lane recognition are compared to a reference

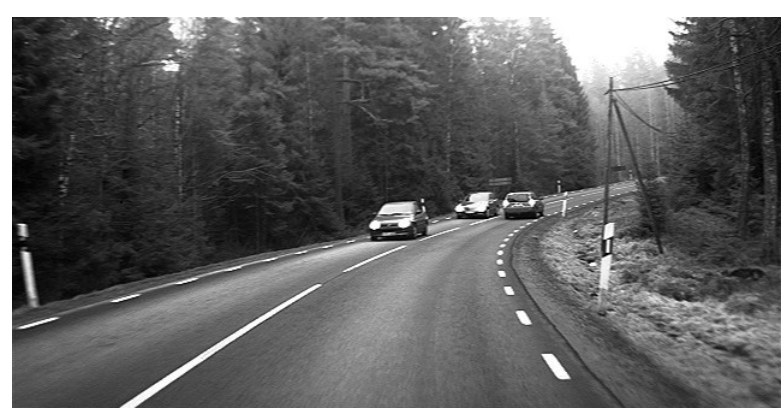

(a)

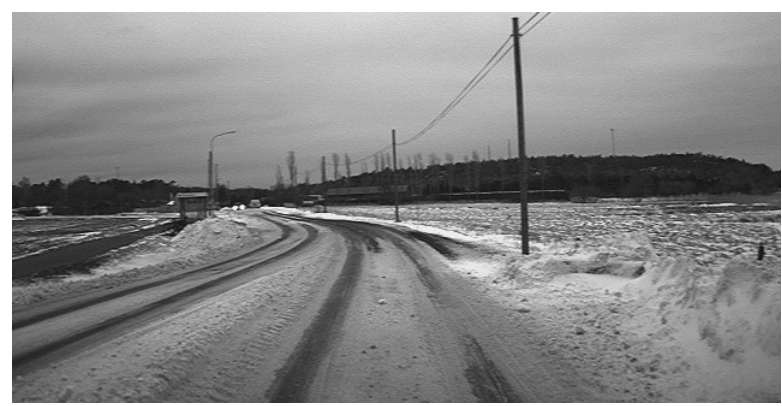

(b)

Figure 8: Two different camera views are shown. In (a) the lane markings are excellent and the leading vehicles are close and clearly visible. This is the traffic situation at $32 \mathrm{~s}$ in the Figures 5 to 7 . Although the circumstances seem perfect, the OLR, Fusion 2 and 3 have problems estimating the curvature, as seen in Figure 7. The traffic situation shown in (b) is more demanding, mainly due to the weather conditions and large distance to the leading vehicle.

value. The reference value is computed off-line using a geometric method described in [51], which applies a least square curve fitting to a sliding window. The entire data set i.e., also future values of the ego vehicle movement, is used to derive the reference value. The accuracy of the method was validated on a test track, where the ground truth is well defined, and the results are good as reported in [51].

A typical result of a comparison is shown in Figure 7. The data stems from a rural road, which explains the curvature values. It can be seen that the estimates from the sensor fusion approaches give better results than using the OLR alone, as was expected. The OLR estimate is rather noisy compared to the fused estimates. This is not surprising, since the raw OLR has less information. A camera view from the curve at time $32 \mathrm{~s}$ is shown in Figure 8a. 


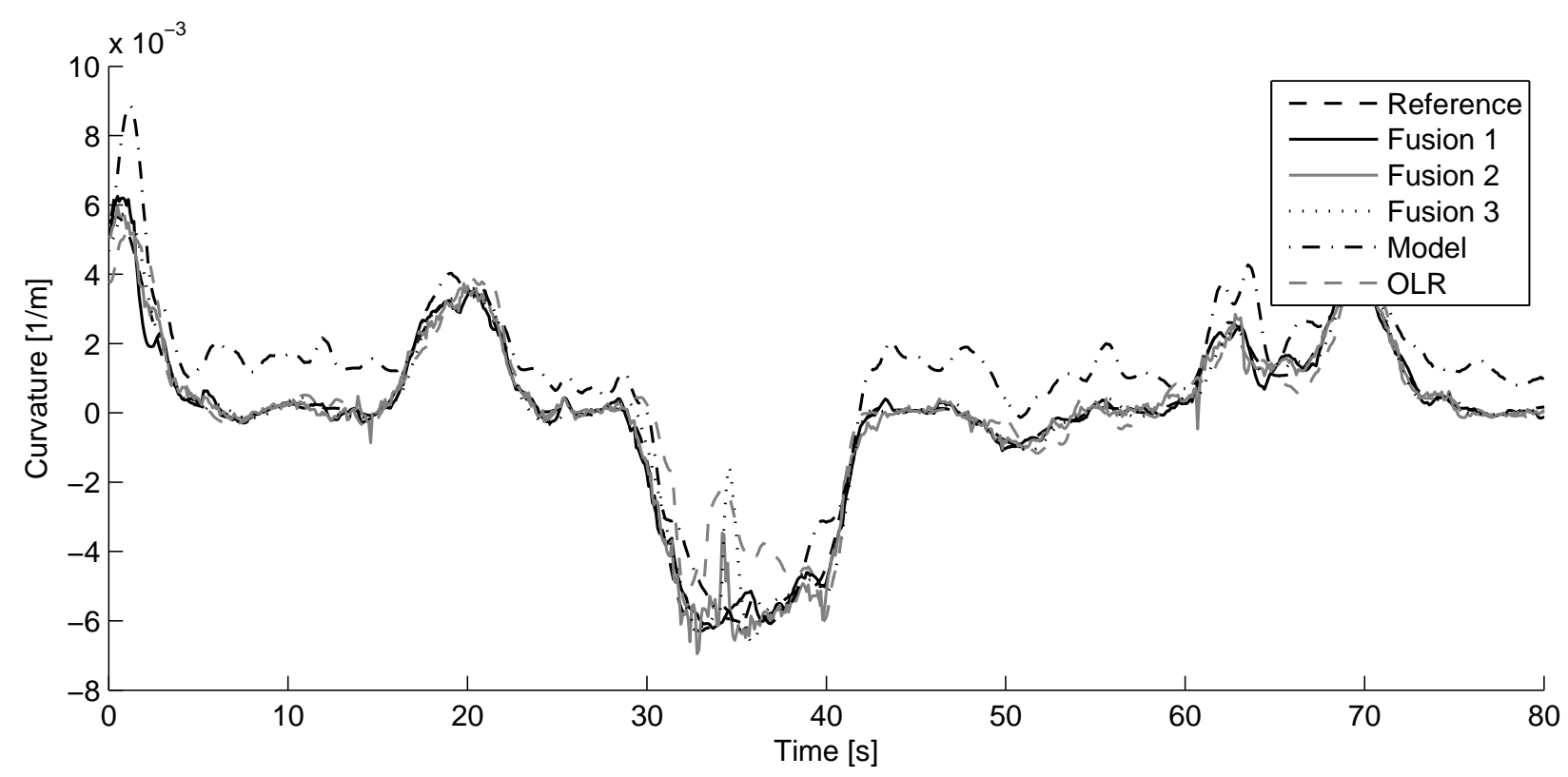

Figure 7: Results from the three fusion approaches (fusion 1 solid black line, fusion 2 gray line and fusion 3 dotted line) and the OLR (dashed gray line), showing the curvature estimate $\hat{c}_{0}$. As can be seen, the curvature estimate can be improved by taking the other vehicles (gray line) and the ego vehicle's driven curvature in to account (solid black line). The model (dash-dotted) is estimating the derivative of the curvature and the absolute position is not measured, which leads to the illustrated bias. The dashed line is the reference curvature.

The curvature estimate from the state-space model described in this paper is denoted by model and is shown as a dash-dotted black line. The absolute position is not measured, which leads to a clearly visible bias in the estimate of $c_{0}$. The bias is transparent in Figure 7, but it also leads to a large RMSE value in Table 1. Fusion 3 also delivers a decent result, but it is interesting to notice that the estimate seems to follow the incorrect OLR at time $35 \mathrm{~s}$. The same behavior holds for fusion 2 in Figure 7.

To get a more aggregate view of the performance, we provide the root mean square error (RMSE) for longer measurement sequences in Table 1. The fusion approaches improve the road curvature estimate by making use of the information about the leading vehicles, that is available from the radar and the vision systems. However, since we are interested in the curvature estimate also when there are no leading vehicles in front of the ego vehicle, this case will be studied as well. It is straightforward to study this case, it is just a matter of not providing the measurements of the leading vehicles to the algorithms. The RMSE values found without information about the leading vehicles are given in the columns marked no in Table 1.
These results should ideally be compared to data where information about the leading vehicles is considered, but during the $78 \mathrm{~min}$ drive there were not always another car in front of us. Only for about $50 \%$ of the time there existed other vehicles, which we could track. Hence, for the sake of comparability we give the RMSE values for those sequences where at least one leading vehicle was tracked, bearing in mind that these are based on only about $50 \%$ of the data. The corresponding columns in Table 1 are marked only. Finally, we also give the RMSE values for the complete data, where other vehicles were considered whenever possible.

It is interesting to see that the advantage of fusion 1, which uses a more accurate ego vehicle and road model, in comparison to fusion 2 is particularly noticeable when driving alone on a rural road, the RMSE for fusion 1 is then 1.18 , whereas the RMSE for fusion 2 is 2.91. The reason for this is first of all that we are driving on a rather curvy road which implies that any additional information will help improving the curvature estimate. Here, the additional information is the improved ego vehicle and road models used in fusion 1. Furthermore, the fact that there are no leading vehicles that could aid the fusion algorithm when driving alone 
Table 1: Comparison of the root mean square error (RMSE) of the road curvature $c_{0}$ in $[1 / m]$ for the three fusion approaches and the pure measurement signal OLR for two longer measurement sequences acquired on public roads in Sweden. Three cases were considered, using only those measurements where a leading vehicle could be tracked, using the knowledge of the leading vehicles position whenever possible or not at all and thereby simulating the lonely driver. Note that all RMSE values should be multiplied by $10^{-3}$.

\begin{tabular}{|c|c|c|c|c|c|c|}
\hline & \multicolumn{3}{|c|}{ Highway } & \multicolumn{3}{|c|}{ Rural road } \\
\hline Time & \multicolumn{3}{|c|}{$44 \min$} & \multicolumn{3}{|c|}{$34 \mathrm{~min}$} \\
\hline OLR $\left[10^{-3} / m\right]$ & \multicolumn{3}{|c|}{0.385} & \multicolumn{3}{|c|}{3.60} \\
\hline Model $\left[10^{-3} / \mathrm{m}\right]$ & \multicolumn{3}{|c|}{0.356} & \multicolumn{3}{|c|}{2.10} \\
\hline Leading vehicles used? & only & possible & no & only & possible & no \\
\hline Fusion $1\left[10^{-3} / \mathrm{m}\right]$ & 0.176 & 0.184 & 0.189 & 1.48 & 1.13 & 1.18 \\
\hline Fusion $2\left[10^{-3} / \mathrm{m}\right]$ & 0.231 & 0.228 & 0.230 & 1.53 & 2.84 & 2.91 \\
\hline Fusion $3\left[10^{-3} / \mathrm{m}\right]$ & 0.203 & 0.210 & 0.205 & 1.32 & 2.01 & 1.94 \\
\hline
\end{tabular}

creates a greater disadvantage for fusion 2 , since it is its main additional information. Fusion 3, which uses the single track vehicle model of fusion 1 , but the road model of fusion 2, seems to position itself between those two.

Comparing the rural road results based only on those measurements where other vehicles were tracked, we see an interesting pattern. The curvature estimate of fusion 2 and fusion 3 is improved by the additional information, but the estimate of fusion 1 is declined. The error values of the three fusion approaches are also in the same range. The explanation of this behavior can be found by analyzing the measurement sequences. If the leading vehicle is close-by, as for example in Figure $8 \mathrm{a}$, it helps improving the results. However, if the leading vehicle is more distant, the curvature at this position might not be the same as it is at the ego vehicle's position, which leads to a degraded result. In [53] the authors presented preliminary results based on much shorter measurement sequences, where the leading vehicles were more close-by and the estimate of fusion 1 was improved by the existence of leading vehicles. The problem could be solved by letting the measurement noise $\mathbf{e}$ of the measurement equation (51) depend on the distance to the leading vehicle.

The highway is rather straight and as expected not much accuracy could be gained in using an improved dynamic vehicle model. It is worth noticing that the OLR's rural road RMSE value is about 10 times higher than the highway value, but the model's RMSE increases only about six times when comparing the rural road values with the highway. Comparing the RMSE values in the columns marked possible; the RMSE for fusion 1 also increases about six times, but that of fusion 2 increases as much as twelve times when compar- ing the highway measurements with the rural road.

A common problem with these road estimation methods is that it is hard to distinguish between the case when the leading vehicle is entering a curve and the case when the leading vehicle is performing a lane change. With the approach in this paper the information about the ego vehicle motion, the OLR and the leading vehicles is weighted together in order to form an estimate of the road curvature. The fusion approach in this paper produces an estimate of the lateral position $l_{T_{n}}$ of the leading vehicle which seems reasonable. The results are thoroughly described in [53].

\section{Conclusions}

In this contribution we have derived a method for joint ego-motion and road geometry estimation. The presented sensor fusion approach combines the information from sensors present in modern premium cars, such as radar, camera and IMU, with a dynamic model. This model, which consists of a new dynamic motion model of the road, is the core of this contribution. The road geometry is estimated by considering the information from the optical lane recognition of the camera, the position of the leading vehicles, obtained by the radar and the camera, and by making use of a dynamic ego vehicle motion model, which takes IMU-data and the steering wheel angle as input. If one of these three parts fails, for example there might not be any leading vehicles or the lane markings are bad, as in Figure 8b, then the sensor fusion framework will still deliver an estimate.

The presented sensor fusion framework has been evaluated together with two other fusion approaches on real and relevant data from both highway and rural roads 
in Sweden. The data consists of 78 min driving on various road conditions, also including snow-covered pavement. The approach presented in this paper obtained the best results in all situations, when compared to the other approaches, but it is most prominent when driving alone on a rural road. If there are no leading vehicles that can be used, the improved road and ego vehicle models still supports the road geometry estimation and delivers a more accurate result.

\section{Acknowledgement}

The authors would like to thank Dr. Andreas Eidehall at Volvo Car Corporation for fruitful discussions. Furthermore, they would like to thank the SEnsor Fusion for Safety (SEFS) project within the Intelligent Vehicle Safety Systems (IVSS) program and the strategic research center MOVIII, funded by the Swedish Foundation for Strategic Research (SSF) for financial support.

\section{Appendix}

Lower case letters are used to denote scalar variables, bold lower case letters are used for vector valued variables and upper case letters are used for matrix valued variables. A superscript letter is used to denote the coordinate frame, in which a variable or constant is represented.

\begin{tabular}{|c|c|}
\hline Abbr. & Explenation \\
\hline$c_{0}$ & road curvature \\
\hline$d$ & a distance \\
\hline$d_{E W}^{W}$ & line from $O_{W}$ to $O_{E}$, in the $W$-frame \\
\hline$\delta_{r}$ & $\begin{array}{l}\text { angle between the vehicle's long. axis and } \\
\text { the lane }\end{array}$ \\
\hline$\delta_{R}$ & $\begin{array}{l}\text { angle between the vehicle's velocity vec- } \\
\text { tor and the lane }\end{array}$ \\
\hline$\delta_{f}$ & mean front wheel angle \\
\hline$\delta_{V_{n}}$ & $\begin{array}{l}\text { azimuth angle between ego vehicle and } \\
\text { leading vehicle }\end{array}$ \\
\hline E & ego vehicle coordinate frame \\
\hline $\mathrm{E}$ & ego model \\
\hline $\mathbf{e}$ & measurement noise \\
\hline$l_{R}$ & $\begin{array}{l}\text { offset between the ego vehicle and the left } \\
\text { lane marking }\end{array}$ \\
\hline$l_{f}$ & $\begin{array}{l}\text { distance between ego vehicle } \mathrm{CoG} \text { and } \\
\text { front axle }\end{array}$ \\
\hline$l_{r}$ & $\begin{array}{l}\text { distance between ego vehicle } \mathrm{CoG} \text { and } \\
\text { rear axle }\end{array}$ \\
\hline
\end{tabular}

\begin{tabular}{|c|c|}
\hline Abbr. & Explenation \\
\hline$l_{s}$ & $\begin{array}{l}\text { distance between ego vehicle CoG and } \\
\text { sensors }\end{array}$ \\
\hline$l_{V}$ & $\begin{array}{l}\text { range between ego vehicle radar and lead- } \\
\text { ing vehicle }\end{array}$ \\
\hline$l_{T}$ & $\begin{array}{l}\text { lateral distance between leading vehicle } \\
\text { and lane marking }\end{array}$ \\
\hline$O_{E}$ & $\begin{array}{l}\text { origin of } E \text {, at the vehicle's center of grav- } \\
\text { ity }\end{array}$ \\
\hline$O_{W}$ & origin of $W$ \\
\hline$P$ & state covariance \\
\hline$\psi_{E}$ & the ego vehicle's yaw angle \\
\hline$Q$ & process noise covariance \\
\hline$R$ & rotation matrix \\
\hline$R$ & measurement noise covariance \\
\hline$R$ & road coordinate frame \\
\hline $\mathrm{R}$ & road model \\
\hline$T$ & target coordinate frame \\
\hline $\mathrm{T}$ & target model \\
\hline$V$ & $\begin{array}{l}\text { coordinate frame in sensor pointing at } \\
\text { leading vehicle }\end{array}$ \\
\hline$W$ & world coordinate frame \\
\hline$w$ & road width \\
\hline $\mathbf{w}$ & process noise \\
\hline $\mathbf{x}$ & state vector \\
\hline$x_{E W}^{W}$ & $\begin{array}{l}\text { X-coordinate of a line from } O_{W} \text { to } O_{E} \text {, in } \\
W \text {-frame }\end{array}$ \\
\hline $\mathbf{y}$ & measurement vector \\
\hline$y_{E W}^{W}$ & $\begin{array}{l}\text { y-coordinate of a line from } O_{W} \text { to } O_{E} \text {, in } \\
W \text {-frame }\end{array}$ \\
\hline
\end{tabular}

\section{References}

[1] G. L. Smith, S. F. Schmidt, L. A. McGee, Application of statistical filter theory to the optimal estimation of position and velocity on board a circumlunar vehicle, Tech. Rep. TR R-135, NASA (1962).

[2] S. F. Schmidt, Application of state-space methods to navigation problems, Advances in Control Systems 3 (1966) 293-340.

[3] B. D. O. Anderson, J. B. Moore, Optimal Filtering, Information and system science series, Prentice Hall, Englewood Cliffs, NJ, USA, 1979.

[4] E. D. Dickmanns, A. Zapp, A curvature-based scheme for improving road vehicle guidance by computer vision, in: Proceedings of the SPIE Conference on Mobile Robots, Vol. 727, Cambridge, MA, USA, 1986, pp. 161-198.

[5] E. D. Dickmanns, B. D. Mysliwetz, Recursive 3-D road and relative ego-state recognition, IEEE Transactions on pattern analysis and machine intelligence 14 (2) (1992) 199-213.

[6] J. C. McCall, M. M. Trivedi, Video-based lane estimation and tracking for driver assistance: Survey, system, and evaluation, IEEE Transactions on Intelligent Transportation Systems 7 (1) (2006) 20-37.

[7] E. D. Dickmanns, Dynamic Vision for Perception and Control of Motion, Springer, London, UK, 2007.

[8] Z. Zomotor, U. Franke, Sensor fusion for improved vision based lane recognition and object tracking with range-finders, in: Pro- 
ceedings of IEEE Conference on Intelligent Transportation System, Boston, MA, USA, 1997, pp. 595-600.

[9] A. Gern, U. Franke, P. Levi, Advanced lane recognition - fusing vision and radar, in: Proceedings of the IEEE Intelligent Vehicles Symposium, Dearborn, MI, USA, 2000, pp. 45-51.

[10] A. Gern, U. Franke, P. Levi, Robust vehicle tracking fusing radar and vision, in: Proceedings of the international conference of multisensor fusion and integration for intelligent systems, Baden-Baden, Germany, 2001, pp. 323-328.

[11] A. Eidehall, J. Pohl, F. Gustafsson, Joint road geometry estimation and vehicle tracking, Control Engineering Practice 15 (12) (2007) 1484-1494.

[12] A. Eidehall, Tracking and threat assessment for automotive collision avoidance, Phd thesis No 1066, Linköping Studies in Science and Technology, SE-581 83 Linköping, Sweden (Jan. 2007).

[13] U. Hofmann, A. Rieder, E. Dickmanns, Ems-vision: application to hybrid adaptive cruise control, in: Proceedings of the IEEE Intelligent Vehicles Symposium, Dearborn, MI, USA, 2000, pp. 468-473.

[14] U. Hofmann, A. Rieder, E. Dickmanns, Radar and vision data fusion for hybrid adaptive cruise control on highways., Machine Vision and Applications 14 (1) (2003) 42 - 49.

[15] R. Schubert, G. Wanielik, K. Schulze, An analysis of synergy effects in an omnidirectional modular perception system, in: Proceedings of the IEEE Intelligent Vehicles Symposium, Xi'an, China, 2009, pp. 54-59.

[16] H. Weigel, P. Lindner, G. Wanielik, Vehicle tracking with lane assignment by camera and Lidar sensor fusion, in: Proceedings of the IEEE Intelligent Vehicles Symposium, Xi' an, China, 2009, pp. 513-520.

[17] A. Muller, M. Manz, M. Himmelsbach, H. Wunsche, A modelbased object following system, in: Proceedings of the IEEE Intelligent Vehicles Symposium, Xi'an, China, 2009, pp. 242249.

[18] H. Loose, U. Franke, C. Stiller, Kalman particle filter for lane recognition on rural roads, in: Proceedings of the IEEE Intelligent Vehicles Symposium, Xi'an, China, 2009, pp. 60-65.

[19] A. Watanabe, T. Naito, Y. Ninomiya, Lane detection with roadside structure using on-board monocular camera, in: Proceedings of the IEEE Intelligent Vehicles Symposium, Xi' an, China, 2009, pp. 191-196.

[20] T. Gumpp, D. Nienhuser, R. Liebig, J. Zollner, Recognition and tracking of temporary lanes in motorway construction sites, in: Proceedings of the IEEE Intelligent Vehicles Symposium, Xi'an, China, 2009, pp. 305-310.

[21] A. Wedel, U. Franke, H. Badino, D. Cremers, B-spline modeling of road surfaces for freespace estimation, in: Proceedings of the IEEE Intelligent Vehicles Symposium, Eindhoven, The Netherlands, 2008, pp. 828-833.

[22] K. Kaliyaperumal, S. Lakshmanan, K. Kluge, An algorithm for detecting roads and obstacles in radar images, Transactions on Vehicular Technology 50 (1) (2001) 170-182.

[23] S. Lakshmanan, K. Kaliyaperumal, K. Kluge, Lexluther: an algorithm for detecting roads and obstacles in radar images, in: Proceedings of the IEEE Conference on Intelligent Transportation System, Boston, MA, USA, 1997, pp. 415-420.

[24] M. Nikolova, A. Hero, Segmentation of a road from a vehiclemounted radar and accuracy of the estimation, in: Proceedings of the IEEE Intelligent Vehicles Symposium, Dearborn, MI, USA, 2000, pp. 284-289.

[25] B. Ma, S. Lakshmanan, A. Hero, Simultaneous detection of lane and pavement boundaries using model-based multisensor fusion, IEEE Transactions on Intelligent Transportation Systems 1 (3) (2000) 135-147.
[26] W. S. Wijesoma, K. R. S. Kodagoda, A. P. Balasuriya, Roadboundary detection and tracking using ladar sensing, IEEE Transactions on Robotics and Automation 20 (3) (2004) 456464.

[27] A. Kirchner, T. Heinrich, Model based detection of road boundaries with a laser scanner, in: Proceedings of the IEEE Intelligent Vehicles Symposium, Stuttgart, Germany, 1998, pp. 93-98.

[28] A. Kirchner, C. Ameling, Integrated obstacle and road tracking using a laser scanner, in: Proceedings of the IEEE Intelligent Vehicles Symposium, Dearborn, MI, USA, 2000, pp. 675-681.

[29] J. Sparbert, K. Dietmayer, D. Streller, Lane detection and street type classification using laser range images, in: Proceedings of the IEEE Intelligent Transportation Systems Conference, Oakland, CA, USA, 2001, pp. 454-459.

[30] C. Lundquist, U. Orguner, T. B. Schön, Tracking stationary extended objects for road mapping using radar measurements, in: Proceedings of the IEEE Intelligent Vehicle Symposium, Xi' an, China, 2009, pp. 405-410.

[31] A. H. Jazwinski, Stochastic processes and filtering theory, Mathematics in science and engineering, Academic Press, New York, USA, 1970.

[32] R. E. Kalman, A new approach to linear filtering and prediction problems, Transactions of the ASME, Journal of Basic Engineering 82 (1960) 35-45.

[33] T. B. Schön, Estimation of nonlinear dynamic systems - theory and applications, Phd thesis No 998, Linköping Studies in Science and Technology, Department of Electrical Engineering, Linköping University, Sweden (Feb. 2006).

[34] T. Kailath, A. H. Sayed, B. Hassibi, Linear Estimation, Information and System Sciences Series, Prentice Hall, Upper Saddle River, NJ, USA, 2000.

[35] N. J. Gordon, D. J. Salmond, A. F. M. Smith, Novel approach to nonlinear/non-Gaussian Bayesian state estimation, in: IEE Proceedings on Radar and Signal Processing, Vol. 140, 1993, pp. 107-113.

[36] F. Bengtsson, L. Danielsson, Designing a real time sensor data fusion system with application to automotive safety, in: Proceedings of the 15 th World Congress of ITS, New York, USA, 2008.

[37] S. S. Blackman, R. Popoli, Design and Analysis of Modern Tracking Systems, Artech House, Inc., Norwood, MA, USA, 1999.

[38] Y. Bar-Shalom, X. R. Li, T. Kirubarajan, Estimation with Applications to Tracking and Navigation, John Wiley \& Sons, New York, 2001.

[39] H. Hahn, Rigid body dynamics of mechanisms. 1, Theoretical basis, Vol. 1, Springer, Berlin, Germany, 2002.

[40] M. Mitschke, H. Wallentowitz, Dynamik der Kraftfahrzeuge, 4th Edition, Springer, Berlin, Heidelberg, 2004.

[41] J. Wong, Theory Of Ground Vehicles, 3rd Edition, John Wiley \& Sons, New York, USA, 2001.

[42] Robert Bosch, GmbH. (Ed.), Automotive Handbook, 6th Edition, SAE Society of Automotive Engineers, 2004.

[43] U. Kiencke, L. Nielsen, Automotive Control Systems, 2nd Edition, Springer, Berlin, Heidelberg, Germany, 2005.

[44] C. Lundquist, T. B. Schön, Recursive identification of cornering stiffness parameters for an enhanced single track model, in: Proceedings of the IFAC Symposium on System Identification, Saint-Malo, France, 2009, pp. 1726-1731.

[45] B. B. Litkouhi, A. Y. Lee, D. B. Craig, Estimator and controller design for lanetrak, a vision-based automatic vehicle steering system, in: Proceedings of the IEEE Conference on Decision and Control, San Antonio, Texas, USA, 1993, pp. 1868 - 1873.

[46] F. Gustafsson, Adaptive Filtering and Change Detection, John Wiley \& Sons, New York, USA, 2000. 
[47] W. J. Rugh, Linear System Theory, 2nd Edition, Information and system sciences series, Prentice Hall, Upper Saddle River, NJ, USA, 1996.

[48] B. O. S. Teixeira, J. Chandrasekar, L. A. B. Torres, L. A Aguirre, D. S. Bernstein, State estimation for equalityconstrained linear systems, in: Proceedings of the IEEE Conference on Decision and Control, New Orleans, LA, USA, 2007, pp. 6220-6225

[49] L. Ljung, System identification, Theory for the user, 2nd Edition, System sciences series, Prentice Hall, Upper Saddle River, NJ, USA, 1999.

[50] C. Lundquist, T. B. Schön, Road geometry estimation and vehicle tracking using a single track model, Tech. Rep. LiTH-ISYR-2844, Department of Electrical Engineering, Linköping University, SE-581 83 Linköping, Sweden (Mar. 2008).

[51] A. Eidehall, F. Gustafsson, Obtaining reference road geometry parameters from recorded sensor data, in: Proceedings of the IEEE Intelligent Vehicles Symposium, Tokyo, Japan, 2006, pp. 256-260.

[52] R. Behringer, Visuelle Erkennung und Interpretation des Fahrspurverlaufes durch Rechnersehen für ein autonomes Straßenfahrzeug, Vol. 310 of Fortschrittsberichte VDI, Reihe 12, VDI Verlag, Düsseldorf, Germany, 1997, also as: PhD Thesis, Universität der Bundeswehr, 1996.

[53] C. Lundquist, T. B. Schön, Road geometry estimation and vehicle tracking using a single track model, in: Proceedings of the IEEE Intelligent Vehicles Symposium, Eindhoven, The Netherlands, 2008, pp. 144-149. 